\title{
Editorial
}

\section{The challenge of alkali metal clusters from the atomic to the nanoscale. Structure, properties and reactivity}

\author{
George Maroulis ${ }^{\mathrm{a}}$ and Patrizia Calaminici ${ }^{\mathrm{b}}$ \\ ${ }^{a}$ Department of Chemistry, University of Patras, Greece \\ E-mail:maroulis@upatras.gr \\ ${ }^{\mathrm{b}}$ Departamento de Quimica, CINVESTAV, Centro De Investigacion Y De Estudios Avanzados, Av. \\ Instituto Politecnico Nacional, 2508, A.P. 14-740, Mexico, D.F. 07000, Mexico \\ E-mail: pcalamin@cinvestav.mx
}

This special issue consists of a collection of papers written by eminent researchers on a broad range of subjects pertaining to alkali metal clusters. The size of the studied systems varies from the atomic level to the nanoscale. The aim and ambition of this special issue is to reach a wide audience of scientists, both experimentalists and theoreticians.

Aguado and López present a study of the melting transition in rotating sodium clusters. Their approach is based on density-functional dymanics simulation. Systems as large as $\mathrm{Na}_{147}$ have been considered.

Martínez et al. consider the reactivity between lithium and the $\mathrm{Al}_{13}$ cluster, relying on density functional theory calculations.

Banerjee and Harbola calculate the $\mathrm{C}_{6}$ van der Waals coefficient for the interaction of sodium clusters with 2-20 atoms. Their approach relies on the jellium model and all-electron ab initio calculations.

Calaminici et al. have presented the diferences of the topology of molecular graphs of small lithium clusters obtained from the density and the molecular electrostatic potential.

The aromaticity of alkali metal clusters has been investigated by Khatua et al.

Dugan and Erkoç have obtained molecular geometries of small lithium clusters using a global optimization technique based on an empirical potential energy function.

An original treatment of superatoms and their assemblies is reported by Clayborne et al. It is shown that stable clusters can mimic the chemical behaviour of atoms. Consequently these molecular architectures can be considered as superatoms that is a third dimension of the periodic table of elements.

Xenides and Maroulis have calculated the (hyper)polarizability of the sodium tetramer.

Nakano and Nozue investigate the orbital degeneracy and magnetic properties of potassium clusters incorporated into nanoporous crystals of zeolite A. This is a complex subject and has rarely been studied before.

Naumkin presents an extensive study of the doping induced structure and property valriations in alkali cluster halides. This original study examines systems as large as $\mathrm{Li}_{13} \mathrm{~F}$ and $\mathrm{Li}_{13} \mathrm{~F}_{2}$. 
The paper by Jiemchooroj et al. reports $\mathrm{C}_{6}$ van der Waals coefficients and polarizabilities for alkali metal clusters and $\mathrm{C}_{60}$. The clusters contain up to ten lithium, potassium or rubidium atoms.

Mohan et al. report a study of a collection of oxo- and sulphur bridged alkali metal centres as MXM with $\mathrm{M}=\mathrm{Li}$, $\mathrm{Na}$ and $\mathrm{K}, \mathrm{X}=\mathrm{O}$ and $\mathrm{S}$.

Zope et al. have calculated dipole polarizabilities for lithium clusters $\mathrm{Li}_{\mathrm{n}}, \mathrm{n} \leqslant 22$, relying on density functional theory calculations.

Guzmań-Ramirez et al. investigated the fragmentation channels of $\mathrm{Na}_{\mathrm{n}}^{+}(\mathrm{n} \leqslant 12)$ cationic clusters. They relied on the widely used B3LYP DFT method to obtain structural and electronic properties.

Iskrenova-Tchoukova et al. have calculated very accurate static polarizabilites for the Cs atom.

Last, Slanina et al. have investigated the molecular structure of molecular architectures involving alkali metal clusters encapsulated into fullerenes.

This collection of papers should be of interest to researchers active in broad fields of modern Chemical Physics, Physical Chemistry and Materials Science. 